(1)

CrossMark

\title{
Traffic exposures, air pollution and outcomes in pulmonary arterial hypertension: a UK cohort study analysis
}

\author{
Eleni Sofianopoulou ${ }^{1,21}$, Stephen Kaptoge ${ }^{1}$, Stefan Gräf (10) $2,3,4$, \\ Charaka Hadinnapola ${ }^{2}$, Carmen M. Treacy ${ }^{2,5}$, Colin Church ${ }^{6,7}$, Gerry Coghlan ${ }^{8}$, \\ J. Simon R. Gibbs ${ }^{9,10}$, Matthias Haimel (102,3,4, Luke S. Howard ${ }^{9,10}$, Martin Johnson ${ }^{6}$, \\ David G. Kiely ${ }^{11}$, Allan Lawrie ${ }^{12}$, James Lordan ${ }^{13}$, Robert V. MacKenzie Ross ${ }^{14}$, \\ Jennifer M. Martin 2,3,4 Shahin Moledina ${ }^{15}$, Michael Newnham², \\ Andrew J. Peacock ${ }^{6}$, Laura C. Price ${ }^{9,16}$, Christopher J. Rhodes ${ }^{17}$, \\ Jay Suntharalingam ${ }^{14}$, Emilia M. Swietlik ${ }^{2,5}$, Mark R. Toshner (1) ${ }^{2,5}$, \\ John Wharton ${ }^{17}$, Martin R. Wilkins ${ }^{17}$, Stephen J. Wort ${ }^{9,16}$, Joanna Pepke-Zaba ${ }^{5}$, \\ Robin Condliffe ${ }^{11}$, Paul A. Corris ${ }^{13}$, Emanuele Di Angelantonio ${ }^{1,18,19}$, \\ Steeve Provencher ${ }^{20}$ and Nicholas W. Morrell $2,4,21$
}

@ERSpublications

In idiopathic pulmonary arterial hypertension, exposure to indirect measures of traffic-related air pollution was associated with haemodynamic severity and ESC/ERS risk score at baseline, whereas exposure to PM2.5 was associated with long-term prognosis http://ow.ly/G8En30o3swc

Cite this article as: Sofianopoulou E, Kaptoge S, Gräf S, et al. Traffic exposures, air pollution and outcomes in pulmonary arterial hypertension: a UK cohort study analysis. Eur Respir J 2019; 53: 1801429 [https://doi.org/10.1183/13993003.01429-2018].

ABSTRACT While traffic and air pollution exposure is associated with increased mortality in numerous diseases, its association with disease severity and outcomes in pulmonary arterial hypertension (PAH) remains unknown.

Exposure to particulate matter with a $50 \%$ cut-off aerodynamic diameter $\leqslant 2.5 \mu \mathrm{m}$ (PM2.5), nitrogen dioxide $\left(\mathrm{NO}_{2}\right)$ and indirect measures of traffic-related air pollution (distance to main road and length of roads within buffer zones surrounding residential addresses) were estimated for 301 patients with idiopathic/heritable PAH recruited in the UK National Cohort Study of Idiopathic and Heritable PAH. Associations with transplant-free survival and pulmonary haemodynamic severity at baseline were assessed, adjusting for confounding variables defined a priori.

Higher estimated exposure to PM2.5 was associated with higher risk of death or lung transplant (unadjusted hazard ratio (HR) $2.68\left(95 \%\right.$ CI 1.11-6.47) per $\left.3 \mu \mathrm{g} \cdot \mathrm{m}^{-3} ; \mathrm{p}=0.028\right)$. This association remained similar when adjusted for potential confounding variables (HR 4.38 (95\% CI 1.44-13.36) per $3 \mu \mathrm{g} \cdot \mathrm{m}^{-3}$; $\mathrm{p}=0.009)$. No associations were found between $\mathrm{NO}_{2}$ exposure or other traffic pollution indicators and transplant-free survival. Conversely, indirect measures of exposure to traffic-related air pollution within the 500-1000 m buffer zones correlated with the European Society of Cardiology/European Respiratory Society risk categories as well as pulmonary haemodynamics at baseline. This association was strongest for pulmonary vascular resistance.

In idiopathic/heritable $\mathrm{PAH}$, indirect measures of exposure to traffic-related air pollution were associated with disease severity at baseline, whereas higher PM2.5 exposure may independently predict shorter transplant-free survival.

This article has supplementary material available from erj.ersjournals.com

Received: Aug 032018 | Accepted after revision: March 022019

Copyright OERS 2019 


\section{Introduction}

Air pollution is an important contributor to premature deaths with over 7 million deaths per year being attributable to traffic and air pollution exposure worldwide [1]. Enhanced exposures to multiple air pollutants, including nitrogen dioxide $\left(\mathrm{NO}_{2}\right)$, sulfur dioxide and fine particulate matter with a $50 \%$ cut-off aerodynamic diameter $\leqslant 2.5 \mu \mathrm{m}(\mathrm{PM} 2.5)$, have been described as independent risk factors for mortality and hospitalisations in the general population $[2,3]$. This has also been observed in specific chronic health conditions, including chronic obstructive pulmonary disease [4, 5], pulmonary fibrosis [6-9] and cardiovascular diseases [10]. Of all of these pollutants, PM2.5 has the greatest effect on human health [2, 11]. Over two-thirds of premature deaths attributable to ambient PM2.5 air pollution are due to cardiovascular disease $[3,12]$. Both acute and chronic exposure to PM2.5 increase the risk of cardiovascular death $[2,13]$, although long-term effects of PM2.5 appear to have a greater impact on cardiovascular mortality [10, 14]. Among cardiovascular events, the occurrence of heart failure exhibited the strongest association with PM2.5 exposure $[13,15]$.

Limited data are available regarding the association between air pollution and outcomes in pulmonary arterial hypertension (PAH), a disease characterised by vasoconstriction and obliterative changes in the small pulmonary arteries, leading to right ventricular failure and death. As for other respiratory diseases, the close relationship between the pulmonary vascular bed and alveolar ventilation makes it highly plausible that air pollution directly affects the pulmonary circulation. Not surprisingly, pre-clinical studies have suggested that prolonged exposure to particulate and soluble antigens can induce changes in pulmonary arteries [16], and that diesel exhaust exposure induces pulmonary hypertension in mice [17]. Imaging studies also found an association between air pollution and changes in right ventricular mass and function within the general population $[18,19]$. As right ventricular function has been repeatedly shown to represent a major determinant of prognosis in PAH [20], we hypothesised that exposure to air pollution is associated with disease severity and outcomes in PAH.

\section{Methods}

\section{Study population and socioeconomic status}

Patients were recruited prospectively from the UK National Cohort Study of Idiopathic and Heritable PAH (www.ipahcohort.com), a multicentre prospective study investigating the genetic and environmental causes of $\mathrm{PAH}$ (details in the supplementary material). Consecutive patients with idiopathic and heritable PAH were then invited to complete a comprehensive epidemiological questionnaire. They were considered eligible if they maintained the same address since diagnosis in order to minimise the risk of exposure misclassification. Potential confounding due to socioeconomic status was addressed by assigning an area-level deprivation to their residential address for each patient using time-weighted Townsend deprivation quantiles that are available for the UK (https://data.mendeley.com/datasets/389scnndjy/1) [21]. Moreover, household income data were collected via questionnaire, taking into account household size and composition [22]. We also accounted for the level of education collected via the epidemiological questionnaire using nine International Standard Classification of Education codes that were collapsed to three categories (Primary/Lower secondary, Upper/Post-secondary and Tertiary) as a measure of socioeconomic status. The Regional Ethics Committee approved this study (REC 13/EE/0203) and participants provided written informed consent. Area-level and

Affiliations: ${ }^{1} \mathrm{MRC} / \mathrm{BHF}$ Cardiovascular Epidemiology Unit, Dept of Public Health and Primary Care, Cardiovascular Epidemiology Unit, University of Cambridge, Cambridge, UK. ${ }^{2}$ Dept of Medicine, University of Cambridge, Cambridge, UK. ${ }^{3}$ Dept of Haematology, University of Cambridge, Cambridge, UK. ${ }^{4} \mathrm{NIHR}$ BioResource - Rare Diseases, Cambridge, UK. ${ }^{5}$ Pulmonary Vascular Diseases Unit, Royal Papworth Hospital, Cambridge, UK. ${ }^{6}$ Scottish Pulmonary Vascular Unit, Regional Heart and Lung Centre, Golden Jubilee National Hospital, Glasgow, UK. ${ }^{7}$ BHF Glasgow Cardiovascular Research Centre, University of Glasgow, Glasgow, UK.

${ }^{8}$ Dept of Cardiology, Royal Free Hospital, London, UK. ${ }^{9}$ National Heart and Lung Institute, Imperial College London, London, UK. ${ }^{10}$ National Pulmonary Hypertension Service, Hammersmith Hospital, London, UK.

${ }^{11}$ Sheffield Pulmonary Vascular Disease Unit, Royal Hallamshire Hospital, Sheffield, UK. ${ }^{12}$ Dept of Infection, Immunity and Cardiovascular Disease, University of Sheffield, Sheffield, UK. ${ }^{13}$ NIHR Biomedical Research Centre in Ageing, University of Newcastle, Newcastle, UK. ${ }^{14}$ National Pulmonary Hypertension Service, Royal United Hospitals Bath NHS Foundation Trust, Bath, UK. ${ }^{15}$ National Paediatric Pulmonary Hypertension Service, Great Ormond Street Hospital, London, UK. ${ }^{16}$ National Pulmonary Hypertension Service, Royal Brompton Hospital, London, UK. ${ }^{17}$ Centre for Pharmacology and Therapeutics, Dept of Medicine, Imperial College London, London, UK. ${ }^{18}$ National Institute for Health Research Blood and Transplant Research Unit in Donor Health and Genomics, Dept of Public Health and Primary Care, University of Cambridge, Cambridge, UK. ${ }^{19} \mathrm{NHS}$ Blood and Transplant, Cambridge, UK. ${ }^{20}$ Pulmonary Hypertension Research Group, Institut Universitaire de Cardiologie et de Pneumologie de Québec Research Center, Laval University, Québec, QC, Canada. ${ }^{21}$ Joint supervision.

Correspondence: Nicholas W. Morrell, Division of Respiratory Medicine, Level 5, Box 157, Addenbrooke's Hospital, Hills Road, Cambridge, CB2 OQQ, UK. E-mail: nwm23Acam.ac.uk 
individual-level deprivation indicators were used in all statistical analyses, given the recognised confounding between deprivation and exposure to air pollution.

\section{Air pollution exposure measures}

Each participant's residential address was assigned a geographic coordinate using QGIS version 2.18 software (https://qgis.org) in conjunction with the UK geocoding database (http://geoconvert.ukdataservice. ac.uk). The geocoded patients' residential addresses were linked to average levels of $\mathrm{PM}_{2.5}$ and $\mathrm{NO}_{2}$ annual means using existing 2010 UK-wide maps (supplementary figure S1), which were based on routine air pollution monitoring data incorporating satellite-derived and chemical transport model estimates plus road and land use data [23]. We assessed associations between mortality as well as disease severity with exposure to increases of $3 \mu \mathrm{g} \cdot \mathrm{m}^{-3}$ for $\mathrm{PM} 2.5$ and $10 \mu \mathrm{g} \cdot \mathrm{m}^{-3}$ for $\mathrm{NO}_{2}$, which correspond to observed interquartile ranges (IQRs) rounded to the nearest integer. We also acquired air pollution monitoring data from the Automatic Urban and Rural Network (https://uk-air.defra.gov.uk/networks/) for the period January 1, 1999 to December 31, 2017. We obtained hourly data for PM2.5 and $\mathrm{NO}_{2}$ from 59 and 73 monitors, respectively, and computed daily averages (supplementary figure S2). To link monitoring data to each residential postal code, we identified the nearest monitoring site within $50 \mathrm{~km}$ of the postal code (based on centroid point) and assigned air pollutant measurements to that postal code. If there were no monitoring sites within the $50 \mathrm{~km}$ zones, we treated the monitored exposure level as missing and excluded that postal code from the analysis. We estimated cumulative air pollution concentrations at lags of $0,3,7$ and 14 days to assess short-term effects of air pollution concentrations on pulmonary haemodynamics at the time of diagnosis. Finally, residential proximity to motorways and A-roads, which form the major road network in the UK, as well as total length of roads within different buffers (100, 200, 500 and $1000 \mathrm{~km})$ around patients' home addresses were estimated as indicators of traffic-related air pollution (supplementary figure S3).

\section{Health outcome measures}

The primary outcome measure was transplant-free survival from time of diagnosis. We also assessed whether markers of air pollution correlated with disease severity, using baseline pulmonary haemodynamic data, i.e. pulmonary vascular resistance, mean pulmonary arterial pressure, cardiac index and right atrial pressure. Finally, we assessed whether markers of air pollution are associated with the abbreviated 2015 European Society of Cardiology (ESC)/European Respiratory Society (ERS) risk stratification strategy, as previously described (supplementary table S1) [24].

\section{Statistical analysis}

Associations of air pollution exposure estimates with risk of death or lung transplantation were assessed based on hazard ratios (HRs) from Cox proportional hazards regression models. In the crude model, we stratified by centre, allowing baseline hazards to be unique for each stratum. In the primary adjustment model, we further adjusted for age, sex and World Health Organization (WHO) functional class. We fitted a further adjusted model accounting for additional confounders including area- and individual-level deprivation, smoking status at diagnosis (current/former/never), season, body mass index, bone morphogenetic protein receptor type II (BMPR2) gene mutation and whether the patient was incident (diagnosed for $<6$ months at study entry) or prevalent. The latter adjustment controlled for any potential survivor bias introduced by the inclusion of prevalent cases in the survival analysis. The covariates were chosen a priori on the basis of potential confounding when assessing health effects of air pollution exposures in PAH. The same covariates were also used in models for disease severity associations.

In survival analysis, we added the ERS/ESC risk category and emphysema as covariates, due to their known effect on mortality as well as data completeness. Given the relatively small number of deaths, we consider the crude and primary adjustment models as reasonable, but caution over interpretation of results from the further adjusted model due to overfitting. To minimise the scope for potential survivor bias due to inclusion of prevalent patients, Cox proportional hazards regression models and survival curves were fitted allowing for left truncation arising from the interval between diagnosis and enrolment. Prevalent patients were only included in the risk set from the time of study entry and were excluded if they entered the study $>10$ years after diagnosis.

Linear mixed effects regression was used to characterise the relationships between air pollution measures and pulmonary haemodynamics, grouping observations by centre. As the haemodynamic outcome variables were natural log-transformed, the estimated regression coefficients (" $\beta$-coefficients") were exponentiated (i.e. $\mathrm{e}^{\beta}$ ) and more naturally interpreted as the expected relative (or percentage) change in outcome. The interpretation of results with outcomes modelled on the logarithmic scale is presented in the supplementary material. The relationship between air pollution measures and ERS/ESC clinical risk prediction tool (low-, intermediate- or high-risk categories) was similarly evaluated, using multinomial 
logistic regression. We excluded the functional class variable from the further adjusted model, as it is required for the estimation of ERS/ESC risk score at baseline.

In order to make the survival analysis models (crude, primary and further adjusted) as comparable as possible, we used a complete-case dataset $(n=286)$ with no missing data of all the variables used for adjustment. We further limited this dataset to a complete-case dataset for haemodynamic variables $(n=243)$ for the disease severity analyses. It was considered unreasonable to conduct multiple imputation analysis as haemodynamics were the health outcomes. The variables of the "nearest monitor" exposure analysis were exempted from the complete-case datasets, due to large numbers of missing records on the air pollution time-series around the patients' diagnosis dates.

When assessing associations with length of road, we accounted for the absence of a major road within the 500-1000 m buffer zones around residences by adding a binary (yes/no) variable on the respective models. We did not model the effect of length of road within the 100-200 m buffer zones as only few participants had a major road within these zones. The traffic exposure indicators were log-transformed. Therefore, log-log regression models were fitted when assessing their association to haemodynamic measures. The exponentiated association estimates were naturally interpreted as expected relative (or percentage) change in haemodynamic outcome for $1 \%$ increase in the traffic exposure indicators. Further information on the interpretation of these models is presented in the supplementary material. Data were presented as means with standard deviation or median (IQR) according to data distribution. Analyses were performed using $\mathrm{R}$ version 3.4.1 (R Project for Statistical Computing, Vienna, Austria) and Stata version 14 (StataCorp, College Station, TX, USA). p-values $<0.05$ were considered as statistically significant.

\section{Results}

Of 537 patients recruited in the UK PAH cohort from January 2014 to February 2018, 406 (76\%) patients were offered and accepted to complete the questionnaire. 100 of them were excluded as they moved since diagnosis and five were excluded as coordinates/addresses were missing, leaving 301 patients for analysis (figure 1). These patients were recruited in seven UK PAH expert centres (supplementary table S2). The noneligible participants were younger and more likely to be prevalent cases (supplementary table S3). Characteristics of the study participants are described in table 1 and were comparable to complete-case datasets used for modelling (supplementary table S4). Table 2 provides summaries of ambient and traffic-related air pollution indicators.

During a mean follow-up of 3.5 years (4.5 years for prevalent cases), 35 (13\%) participants died and five (2\%) were transplanted, resulting in 1-, 3- and 5-year transplant-free survival estimates of $97 \%, 85 \%$ and $67 \%$ for incident patients and $100 \%, 100 \%$ and $88 \%$ for prevalent patients, respectively. The distribution of PM2.5 exposure, among those participants who died compared with those who did not, is presented in supplementary figure S4. Exposure to higher PM2.5 annual (2010 map) mean concentrations was associated with higher risk of death or lung transplant (unadjusted HR 2.68 (95\% CI 1.11-6.47) per $3 \mu \mathrm{g} \cdot \mathrm{m}^{-3} ; \mathrm{p}=0.028$ ) (table 3). Similar estimates were observed when adjusting for potential confounding variables (HR 4.38 (95\% CI 1.44-13.36) per $3 \mu \mathrm{g} \cdot \mathrm{m}^{-3}$; $\left.\mathrm{p}=0.009\right)$. Conversely, no associations were found between recent exposure to PM2.5 at the time of baseline right heart catheterisation (nearest monitoring sites), exposure to $\mathrm{NO}_{2}$ or traffic-related pollution indicators and mortality. However, increases in the sum

FIGURE 1 Flowchart of patient selection. PAH: pulmonary arterial hypertension.

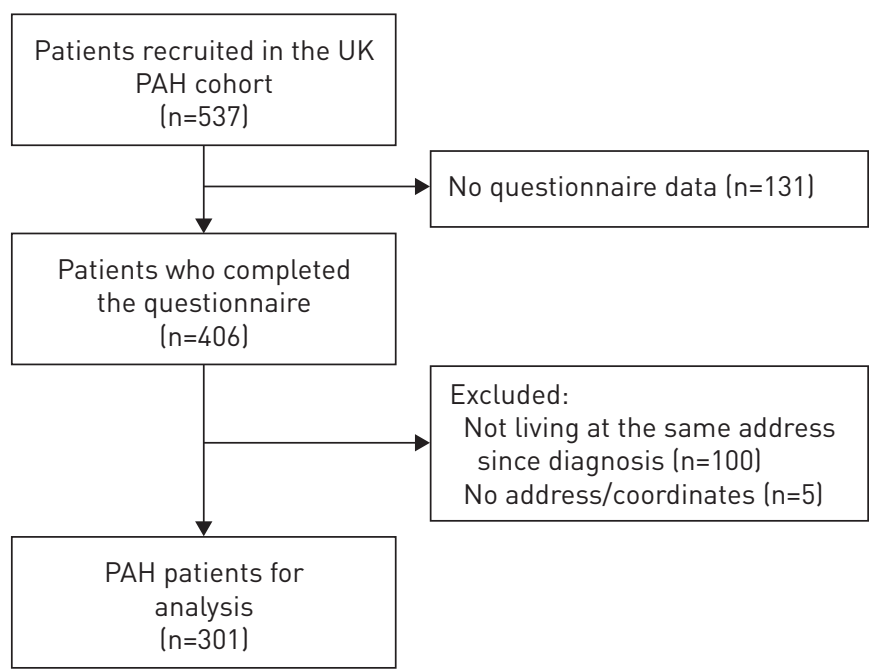




\section{TABLE 1 Characteristics of the study participants at diagnosis}

Subjects
Age year

Female

$51 \pm 15$

PAH subgroup

Idiopathic

$199(66)$

Heritable

WHO functional class

I-II

III

IV

BMI $\mathbf{k g} \cdot \mathrm{m}^{-2}$

Presence of emphysema on CT scan"

Pulmonary haemodynamics

RAP $\mathrm{mmHg}$

mPAP $\mathrm{mmHg}$

$9(7)^{\text {ก1 }}$

Cardiac index $\mathrm{L} \cdot \mathrm{min}^{-1} \cdot \mathrm{m}^{-2}$

Cardiac output L. $\mathrm{min}^{-1}$

PVR WU

PCWP $\mathrm{mmHg}$

$\mathrm{SvO}_{2} \%$

6MWD m

Pulmonary function tests

FEV $1 \%$ pred

Kco \% pred

$84 \pm 19$

Area-level deprivation

Quantile 1 (most deprived)

$74 \pm 24$

Quantile 2

95 (32)

68 (23)

Quantile 3

$61(21)$

Quantile 4

$52(18)$

Quantile 5

Missing category

Household income

Quantile 1

40 (13)

Quantile 2

40 (13)

Quantile 3

$50(17)$

Quantile 4

28 (9)

Quantile 5 (most deprived)

$102(34)$

\section{Education}

Primary/lower secondary

62 (21)

Upper/post-secondary

Tertiary

Missing category

Smoking status at diagnosis

Current smoker

Ex-smoker

Never-smoker

Missing category

Ethnicity

White

Asian

Other $^{+}$

Data are presented as $\mathrm{n}$, mean \pm SD or $\mathrm{n}(\%)$, unless otherwise stated; the percentages may not add up to 100\% due to rounding. PAH: pulmonary arterial hypertension; WHO: World Health Organization; BMI: body mass index; CT: computed tomography; RAP: right atrial pressure; mPAP: mean pulmonary arterial pressure; PVR: pulmonary vascular resistance; WU: Wood units; PCWP: pulmonary capillary wedge pressure; $\mathrm{SvO}_{2}$ : mixed venous oxygen saturation; 6MWD: 6-min walk distance; $\mathrm{FEV}_{1}$ : forced expiratory volume in $1 \mathrm{~s}$; Kco: transfer coefficient of the lung for carbon monoxide. Data were complete for all variables except where indicated by a missing category and further missing data on the continuous variables: $B M I n=9$, RAP $n=16$, cardiac index $n=16$, PAP $n=5$, cardiac output $n=10, P V R n=35, P C W P n=35$, $\mathrm{SvO}_{2} \mathrm{n}=27,6 \mathrm{MWD} \mathrm{n}=92, \mathrm{FEV} 1 \mathrm{n}=23$ and $\mathrm{KCO} \mathrm{n}=46$. . $^{\text {: }}$ the presence of emphysema was based on baseline chest CT at the time of diagnosis; " : median (interquartile range); ${ }^{+}$: includes those of black and mixed ethnicity, and those who preferred not to answer. 


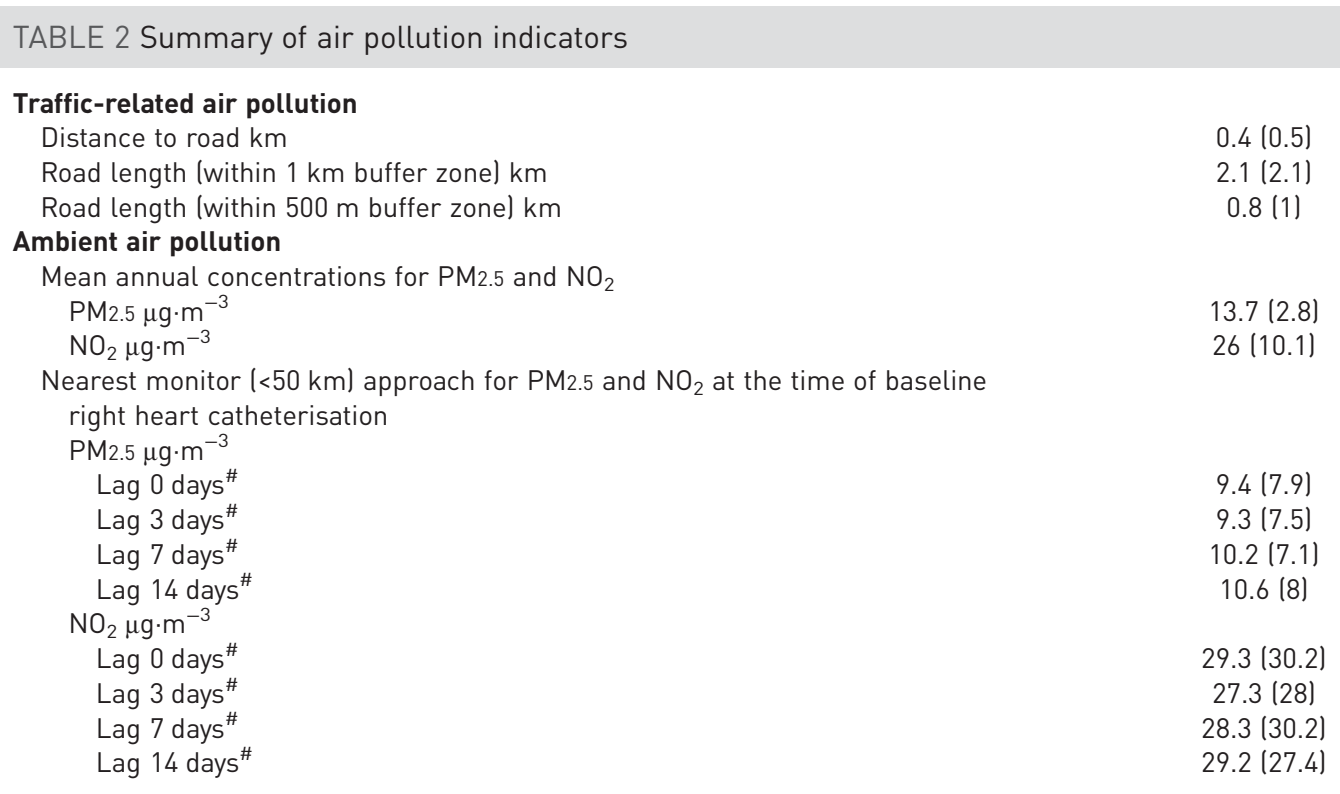

Data are presented as median (interquartile range). PM2.5: particulate matter with a $50 \%$ cut-off aerodynamic diameter $\leqslant 2.5 \mu \mathrm{m} ; \mathrm{NO}_{2}$ : nitrogen dioxide. ${ }^{\#}$ : cumulative air pollution concentrations at lags of $0,3,7$ and 14 days preceding the date of diagnosis.

of the length of main roads within the $500 \mathrm{~m}$ buffer zone were associated with higher PAH risk score at baseline, increasing the likelihood of being diagnosed within the ESC/ERS high-risk clinical prediction category (figure 2).

Baseline haemodynamic severity correlated with distance to main road and the length of road surrounding the residential addresses for both the crude (supplementary figure S5) and the further adjusted (figure 3) models. The strongest evidence for this association was found between pulmonary vascular resistance, mean pulmonary arterial pressure and distance to road indicator, with expected relative change of 0.95 (95\% CI 0.93-0.98; $\mathrm{p}=0.001)$ and 0.98 (95\% CI 0.96-0.99; $\mathrm{p}=0.003$ ) for a $200 \mathrm{~m}$ increase in distance to road, respectively. Surprisingly, increased exposure to PM2.5 (2010 map) was associated with a lower pulmonary vascular resistance (figure 4). No consistent association was found between pulmonary haemodynamics and $\mathrm{NO}_{2}$ (supplementary figures $\mathrm{S} 7$ and $\mathrm{S} 8$ ).

\section{Discussion}

The main findings of this multicentre UK PAH cohort are that direct and indirect measures of exposure to air pollution were associated with more severe disease at baseline and poorer transplant-free survival among patients with idiopathic and heritable PAH. One exception was that increased annual mean PM2.5

TABLE 3 Cox proportional regression analysis for the association between annual (2010) mean concentrations of particulate matter with a $50 \%$ cut-off aerodynamic diameter $\leqslant 2.5 \mu \mathrm{m}$ (PM2.5) exposure and risk of mortality or transplantation: complete dataset for survival analysis ${ }^{\#}$

\begin{tabular}{lcc} 
& Hazard ratio $(95 \% \mathrm{Cl})$ & p-value \\
\hline Crude model $^{\Uparrow}$ & $2.68(1.11-6.47)$ & 0.028 \\
${\text { Primary adjusted } \text { model }^{+}}^{\text {Further adjusted } \text { model }^{\S}}$ & $2.74(1.06-7.05)$ & 0.037 \\
\hline
\end{tabular}

Hazard ratios per $3 \mu \mathrm{g} \cdot \mathrm{m}^{-3}$ increase of PM2.5 exposure. ${ }^{\#}$ : complete dataset with no missing data for the variables we adjusted for in the main (survival analysis) models ( $n=286$ ): due to exclusion criteria related to survival analysis, i.e. prevalent patients who were diagnosed $>10$ years before study entry $(n=16)$ and cases who left before the study initiation ( $n=2)$, the final models comprised of 268 (40 death/transplanted) cases; ๆ: model stratified by centre; ${ }^{+}$: model adjusted for age, sex, World Health Organization functional class, stratified by centre; §: model adjusted for centre, age, sex, deprivation, income, education, smoking, incident/prevalent, emphysema, European Respiratory Society/European Society of Cardiology risk category and BMPR2 mutation, stratified by centre. 

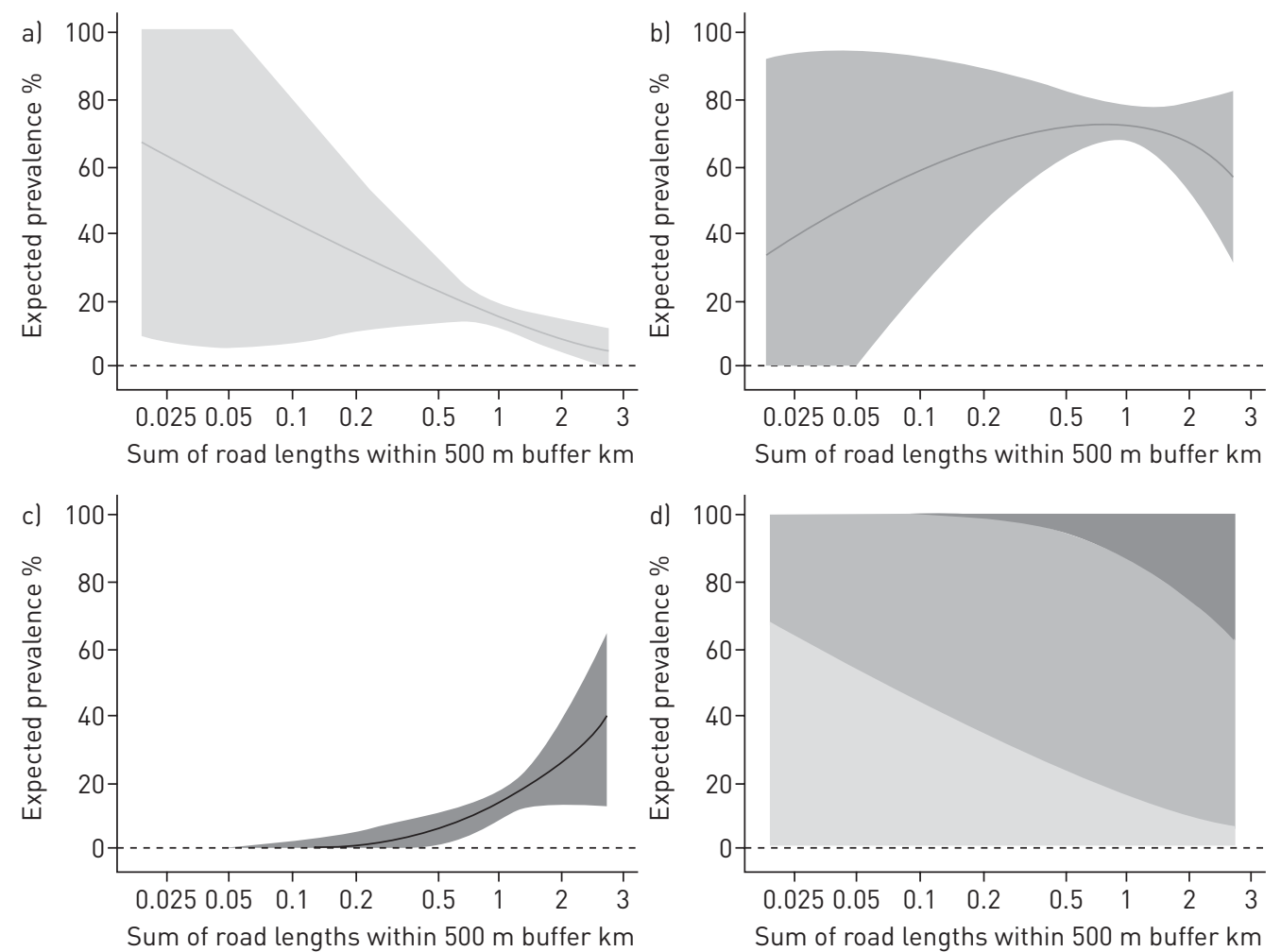

FIGURE 2 Multinomial logistic regression estimating the associations between pulmonary arterial hypertension risk profile and length of main roads within the $500 \mathrm{~m}$ buffer zone. a-c) Expected risk $(95 \% \mathrm{CI})$ of being diagnosed in the a) low-, b) intermediate- and c) high-risk profiles according to the abbreviated European Society of Cardiology (ESC)/European Respiratory Society (ERS) risk score. An increase in length of road was associated with increased probability of having a high risk score at baseline. d) Summary of the association between the sum of road lengths within the $500 \mathrm{~m}$ buffer zone and the risk of being within the low-, medium- and high-risk ESC/ERS risk categories at diagnosis. The shading in (d) corresponds to the shading used in (a)-(c).

concentrations were associated with lower pulmonary vascular resistance. While estimated exposure to PM2.5 was independently associated with the risk of death or transplantation, traffic air pollution indicators at the time of diagnosis correlated with the ESC/ERS risk stratification and baseline haemodynamic severity. These associations should, however, be interpreted with caution given the limited sample size and the indirect measures used as proxy for air pollution exposure.

Increased air pollution exposure has been documented as an independent risk factor for mortality and hospitalisations in the general population [2,3], and in several chronic health conditions [4-9, 15]. Evidence suggests that these effects are stronger for cardiovascular mortality than for other causes of mortality $[25,26]$. Moreover, PM2.5 emissions have been consistently found to have more detrimental health effects compared with gaseous emissions (e.g. $\mathrm{NO}_{2}$ ), with cardiovascular events exhibiting the strongest association with exposure to PM2.5 $[13,15]$. Particles generated by diesel exhaust are considered particularly toxic as they carry a much larger fraction of toxic compounds [10,27]. Particulate matter is a mixture of solid particles and liquid droplets suspended in the air. Contrary to larger particles, PM2.5 have a diameter that enables particles to penetrate deep into the lungs and permeate the alveolar-capillary epithelium [25]. The size of particles is thus directly linked to their impact to influence human health. Numerous biological mechanisms have been hypothesised to explain the association between PM2.5 exposure and cardiovascular diseases. These include the possible direct actions of pollutants reaching the lung and the systemic circulation, contributing to oxidative stress, autonomic imbalance $[10,14]$, epigenetic dysregulation [28], immunomodulation [29] and vascular inflammation [30].

In the Multi-Ethnic Study of Atherosclerosis and Air Pollution (MESA Air), long-term ambient PM2.5 concentrations were shown to be associated with the progression of intima-media thickness on ultrasound examination [31] and ambient air pollution was associated with a greater peripheral total pulmonary vascular volume [32]. Interestingly, experimental studies have documented that prolonged exposure to particulate and soluble antigens induces changes in pulmonary arteries [16], and that diesel exhaust exposure induces pulmonary hypertension in mice [17]. Moreover, an echocardiographic study on 81 


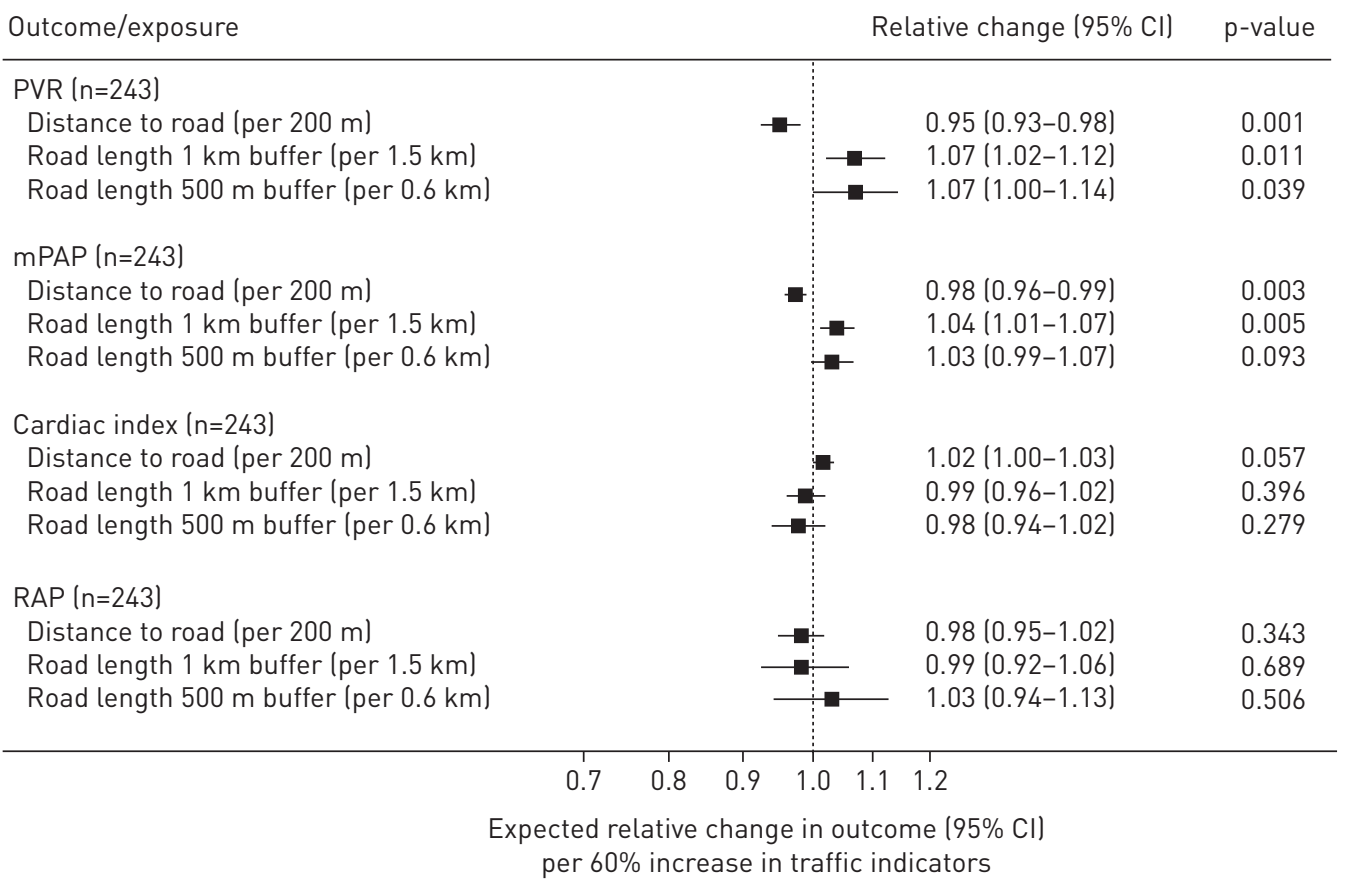

FIGURE 3 Multivariable linear regression estimating the associations between pulmonary haemodynamics and traffic air pollution: pulmonary vascular resistance (PVR; Wood units), mean pulmonary arterial pressure (mPAP; $\mathrm{mmHg}$ ), cardiac index $\left(\mathrm{L} \cdot \mathrm{min}^{-1} \cdot \mathrm{m}^{-2}\right)$ and right atrial pressure (RAP; $\mathrm{mmHg}$ ). The fully adjusted model was adjusted for: age, sex, functional class, smoking status at diagnosis, season, area deprivation, income, education, body mass index, prevalent/incident cases, presence of BMPR2 mutation and centre. Complete-case dataset with no missing data for the haemodynamics and the variables we adjusted for $(n=243)$. Both exposure and haemodynamic variables were log-transformed. Therefore, the relative changes represent percentage change of the haemodynamics for a $60 \%$ increase in the traffic exposure indicators. A $60 \%$ increase in the geometric mean of traffic exposure indicators approximates to $200 \mathrm{~m}$ increases for distance to road, $0.6 \mathrm{~km}$ increases for road length within $500 \mathrm{~m}$ buffer zone and $1.5 \mathrm{~km}$ increases for road length within $1 \mathrm{~km}$ buffer zone.

children living in Mexico also found that outdoor exposure levels of PM2.5 were associated with increased pulmonary arterial pressure and with elevated plasma endothelin-1 levels [33], while a chamber study on 18 healthy volunteers showed that diesel exhaust exposure increases pulmonary vascular resistance at high cardiac output [34]. In view of the small numbers of subjects involved in these studies and the limited pre-clinical experiments on the pulmonary circulation physiology in response to exposure to pollutants, the mechanisms underlying the association between indirect measures of air traffic pollution and haemodynamic severity at baseline in the present study remain elusive. Nonetheless, we can speculate that air pollution could contribute to enhanced inflammation, oxidative stress and endothelial dysfunction that have been involved in PAH development and progression.

Contrary to the scant evidence on the association between pulmonary vascular dysfunction and air pollution in humans, multiple studies confirmed the association between air pollution exposure and heart structure and function. The MESA study confirmed that people living in proximity to roadways had greater left ventricular mass [35]. More recently, the MESA study also confirmed that exposure to higher concentrations of $\mathrm{NO}_{2}$ [36], PM2.5 [37] and PM2.5-10 [19] were associated with greater right ventricular mass and right ventricular end-diastolic volume. The association between chronic past exposure to traffic-related pollutants and right ventricular and left ventricular mass and function were also recently replicated within the UK Biobank Population Imaging Study [18]. Even acute increases in PM2.5 exposure were recently shown to induce endothelial dysfunction and influence cardiac function [38]. As the prognosis of PAH is mostly influenced by the consequences of the increased afterload on right heart function, the present association between air pollution exposure and PAH outcomes is therefore not unexpected.

To the best of our knowledge, this is the first study that aimed at investigating the association between traffic-related and air pollution exposure and haemodynamic severity and outcomes in PAH. We found strong evidence for the association between pulmonary vascular resistance and distance to the nearest main road. Importantly, despite being exposed at PM2.5 concentrations below current air quality standards, PM2.5 exposure was associated with increases in the relative risk of mortality. Moreover, this association was robust using different model specifications. Whether this finding is related to influences of pollutant 


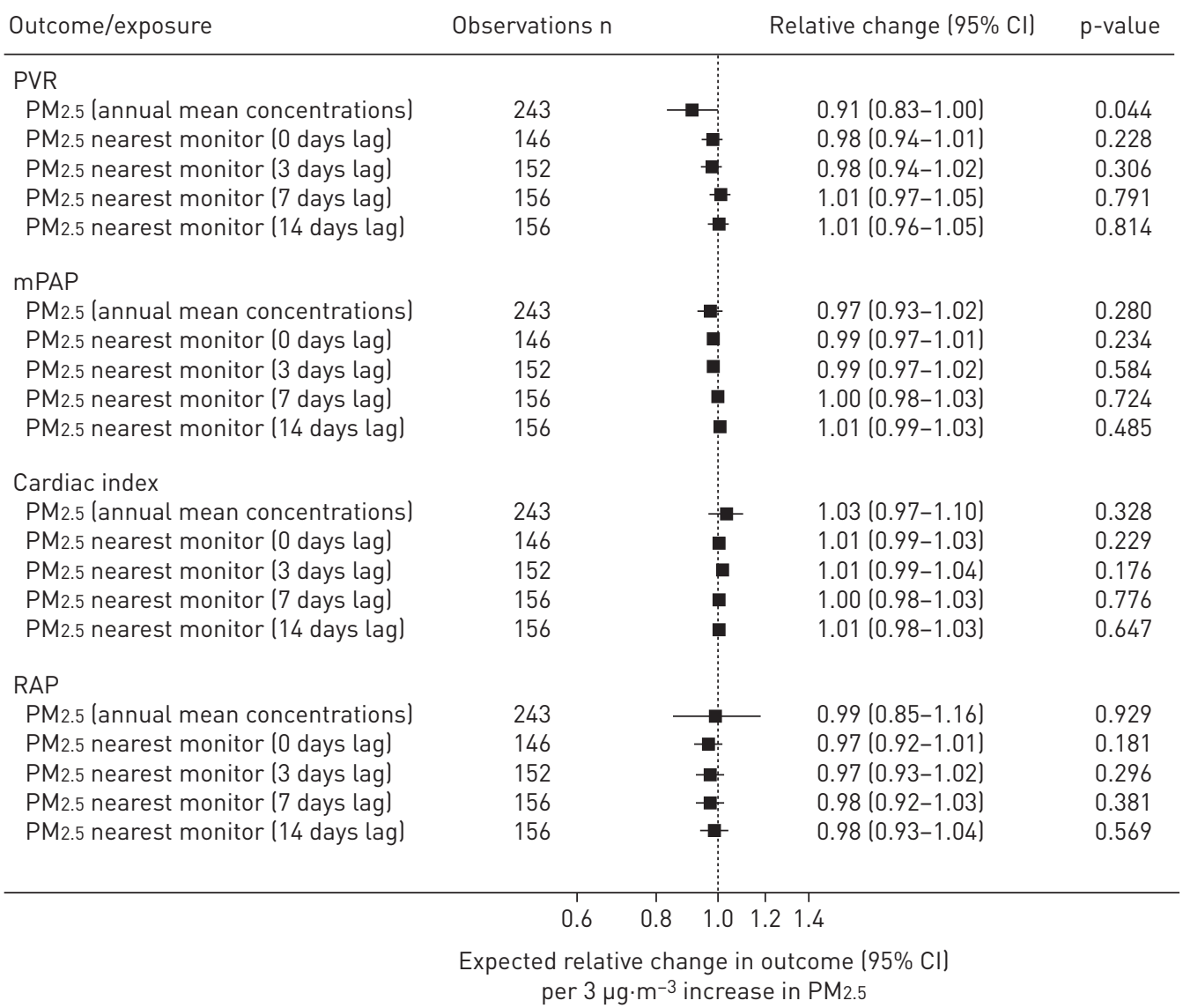

FIGURE 4 Multivariable linear regression estimating the associations between pulmonary haemodynamics and particulate matter with a $50 \%$ cut-off aerodynamic diameter $\leqslant 2.5 \mu \mathrm{m}$ (PM2.5) concentrations: pulmonary vascular resistance (PVR; Wood units), mean pulmonary arterial pressure (mPAP; $\mathrm{mmHg}$ ), cardiac index $\left(\mathrm{L} \cdot \mathrm{min}^{-1} \cdot \mathrm{m}^{-2}\right)$ and right atrial pressure (RAP; $\left.\mathrm{mmHg}\right)$. The model was fully adjusted for age, sex, functional class, smoking status at diagnosis, season, area deprivation, income, education, body mass index, prevalent/ incident cases, presence of BMPR2 mutation and centre. Only haemodynamic variables were log-transformed. Therefore, the relative changes represent percentage change in haemodynamics per $3 \mu \mathrm{g} \cdot \mathrm{m}^{-3}$ (interquartile range rounded to the nearest integer) increase in PM2.5 exposure.

exposure on right ventricular function, PAH progression, comorbidities or a coincidental finding remains to be investigated. It is noteworthy that the magnitude of this association was higher than reported in most observational studies from the general population in which relative increases in mortality were most commonly in the range of $4-15 \%$ per $10 \mu \mathrm{g} \cdot \mathrm{m}^{-3}$ of PM2.5 concentrations $[2,3]$. Subgroups of the general population can, however, be particularly susceptible to air pollution. In a group of post-menopausal females, a $76 \%$ increase in the risk of death from cardiovascular disease was observed per $10 \mu \mathrm{g} \cdot \mathrm{m}^{-3}$ of PM2.5 concentrations [39]. Although the present study's estimate was imprecise, with wide confidence intervals, it remains nonetheless consistent with the increased risk of death observed in more vulnerable populations [39-41], as with disease-specific mortality [3]. The present results are observed for air pollution levels that meet the current air quality standards, which is also the case for other studies in the USA and Europe $[2,42]$. These observations thus support current efforts to improve outdoor air quality and meet the WHO reference level for PM2.5 annual mean concentrations $\left(10 \mu \mathrm{g} \cdot \mathrm{m}^{-3}\right)$. Intriguingly, previous studies suggested that air pollution exposure correlates not only with pulmonary fibrosis severity but also its incidence [9]. While low disease awareness was proposed to explain the higher prevalence of PAH in urban regions of France in previous registry data [43], the impact of air pollution cannot be fully excluded.

The present study has several limitations that should be acknowledged. As evidence suggests that mean $\mathrm{PM} 2.5$ and $\mathrm{NO}_{2}$ concentrations minimally fluctuate over the years [15], we estimated individual exposure to air pollution using the 2010 annual average air pollution maps of ambient $\mathrm{PM} 2.5$ and $\mathrm{NO}_{2}$ concentrations. We also linked each study participant's residence to the location of the monitoring stations. However, the use of the nearest monitor represents a coarse exposure approach as it does not account for spatial heterogeneity due to land use (i.e. roads) or prevailing winds. Thus, $50 \mathrm{~km}$ can capture large rural areas as well as urban areas. Moreover, people who live in industrialised countries spend $~ 90 \%$ 
of their time indoors [44]. Similarly, even if PAH patients are expected to spend more time at home than the general population, many people may spend much of their time at their workplace, which may be far from their home. In addition, smoking and individual-level deprivation data only partly captured indoor air quality. Thus, available maps are likely to have led to exposure misclassification for many patients of the present cohort. Intriguingly, enhanced PM2.5 exposure using 2010 air pollution maps was associated with poorer survival but lower pulmonary vascular resistance at baseline. The mechanisms underlying these associations remain elusive. It is noteworthy, however, that such discrepancies between specific pollutant exposures and outcomes have been repeatedly observed in other conditions, including chronic obstructive pulmonary disease [4,5] and pulmonary fibrosis [7-9]. Additionally, in order to minimise exposure misclassification, a significant proportion of patients were ineligible as they did not maintain the same address since diagnosis. As moving may not occur randomly, this may have led to selection bias, questioning the external validity of our findings. Moreover, the traffic-related air pollutant exposure has been associated with outcomes in small cohorts of patients with specific cardiorespiratory diseases $[7,8]$. However, the present cohort had a smaller sample size than most observational studies evaluating the association between traffic road or air pollutant exposure and outcomes [2, 42]. Whether the observed associations result from sampling error or reflect the vulnerable nature of patients with PAH remains to be determined. The potential for a false-positive finding also cannot be excluded, as multiple comparisons increase the possibility for type I error in statistical analyses. Finally, as PM2.5 is a heterogeneous mixture of solid and liquid particles emitted from a variety of sources, and traffic-related air pollution indicators are indirect measures of air pollution, the available data precluded the estimation of the specific constituents and sources of the particles. Given these limitations, the current data should be interpreted with caution until larger studies are available.

In conclusion, traffic-related air pollution and PM2.5 exposure may be associated with disease severity and outcomes in PAH. However, our findings require validation and replication in a larger cohort to increase confidence in the reliability of these estimates. The present study thus encourages further investigations on air pollution exposure as a potentially removable risk factor influencing PAH incidence, severity and outcomes.

Acknowledgements: The UK National Cohort of Idiopathic and Heritable PAH is supported by the National Institute for Health Research (NIHR), the British Heart Foundation (BHF) (SP/12/12/29836; RG/13/13/30194; RG/18/13/33946), the BHF Cambridge Centre of Cardiovascular Research Excellence, the UK Medical Research Council (MR/K020919/1; MR/L003120/1), the Dinosaur Trust and the NIHR Cambridge Biomedical Research Centre at the Cambridge University Hospitals NHS Foundation Trust. The views expressed are those of the authors and not necessarily those of the NHS, the NIHR or the Department of Health and Social Care. N.W. Morrell holds a BHF Personal Chair Award and is an NIHR Senior Investigator. We thank all the patients and their families who contributed to this research and the Pulmonary Hypertension Association (UK) for their support.

Conflict of interest: E. Sofianopoulou has nothing to disclose. S. Kaptoge reports grants from UK Medical Research Council and British Heart Foundation, during the conduct of the study. S. Gräf has nothing to disclose. C. Hadinnapola has nothing to disclose. C.M. Treacy has nothing to disclose. C. Church has nothing to disclose. G. Coghlan has nothing to disclose. J.S.R. Gibbs reports grants and personal fees from Actelion, Bayer, GSK and MSD, personal fees from Arena, Bellerophon, Complexa and Pfizer, grants from Amco and United Therapeutics, outside the submitted work. M. Haimel has nothing to disclose. L.S. Howard has nothing to disclose. M. Johnson reports grants and personal fees for attendance at meeting and lectures from Actelion, Bayer, GSK and MSD, outside the submitted work. D.G. Kiely reports grants, personal fees and nonfinancial support from Actelion, Bayer and GSK, personal fees and nonfinancial support from MSD, outside the submitted work. A. Lawrie reports grants and personal fees from Actelion and GSK, grants from British Heart Foundation and UK Medical Research Council, outside the submitted work. J. Lordan has nothing to disclose. R.V. MacKenzie Ross has nothing to disclose. J.M. Martin has nothing to disclose. S. Moledina has nothing to disclose. M. Newnham reports education support (travel, registration and accommodation) to attend conferences from MSD and GSK, outside the submitted work. A.J. Peacock reports grants and personal fees from Actelion and Bayer, personal fees from GSK, grants from Gilead, outside the submitted work. L.C. Price has nothing to disclose. C.J. Rhodes has nothing to disclose. J. Suntharalingam has nothing to disclose. E.M. Swietlik has nothing to disclose. M.R. Toshner has nothing to disclose. J. Wharton reports personal fees for advisory board work from Actelion, outside the submitted work. M.R. Wilkins has nothing to disclose. S.J. Wort reports grants and personal fees from Actelion and Bayer, personal fees from GSK and MSD, outside the submitted work. J. Pepke-Zaba (or her institution) received research, education grants from Actelion, Merck and Bayer; and has served on advisory boards for Actelion, Merck, Bayer and GSK. R. Condliffe reports personal fees for advisory board work from Actelion, Bayer and MSD, during the conduct of the study. P.A. Corris reports grants and personal fees from Actelion and Bayer, personal fees from MSD, outside the submitted work. E. Di Angelantonio reports grants from European Commission Framework 7, European Research Council, British Heart Foundation, UK Medical Research Council and National Institute for Health Research, during the conduct of the study; grants from NHS Blood and Transplant, outside the submitted work. S. Provencher has nothing to disclose. N.W. Morrell reports personal fees from GSK and Johnson \& Johnson/Actelion, outside the submitted work.

Support statement: This work was funded by the British Heart Foundation (RG68204) and the Research Councils UK, Medical Research Council (RG67444). Funding information for this article has been deposited with the Crossref Funder Registry. 


\section{References}

1 World Health Organization. Global health risks: mortality and burden of disease attributable to selected major risks. 2009. www.who.int/healthinfo/global_burden_disease/GlobalHealthRisks_report_full.pdf Date last accessed: June 27, 2018.

2 Di Q, Wang Y, Zanobetti A, et al. Air pollution and mortality in the Medicare population. N Engl J Med 2017; 376: 2513-2522.

3 Beelen R, Raaschou-Nielsen O, Stafoggia M, et al. Effects of long-term exposure to air pollution on natural-cause mortality: an analysis of 22 European cohorts within the multicentre ESCAPE project. Lancet 2014; 383: 785-795.

4 DeVries R, Kriebel D, Sama S. Outdoor air pollution and COPD-related emergency department visits, hospital admissions, and mortality: a meta-analysis. COPD 2017; 14: 113-121.

5 Gan WQ, FitzGerald JM, Carlsten C, et al. Associations of ambient air pollution with chronic obstructive pulmonary disease hospitalization and mortality. Am J Respir Crit Care Med 2013; 187: 721-727.

6 Johannson KA, Vittinghoff E, Lee K, et al. Acute exacerbation of idiopathic pulmonary fibrosis associated with air pollution exposure. Eur Respir J 2014; 43: 1124-1131.

7 Johannson KA, Vittinghoff E, Morisset J, et al. Air pollution exposure is associated with lower lung function, but not changes in lung function, in patients with idiopathic pulmonary fibrosis. Chest 2018; 154: 119-125.

8 Sese L, Nunes $\mathrm{H}$, Cottin V, et al. Role of atmospheric pollution on the natural history of idiopathic pulmonary fibrosis. Thorax 2018; 73: 145-150.

9 Conti S, Harari S, Caminati A, et al. The association between air pollution and the incidence of idiopathic pulmonary fibrosis in Northern Italy. Eur Respir J 2018; 51: 1700397.

10 Newby DE, Mannucci PM, Tell GS, et al. Expert position paper on air pollution and cardiovascular disease. Eur Heart J 2015; 36: 83-93.

11 Cesaroni G, Forastiere F, Stafoggia M, et al. Long term exposure to ambient air pollution and incidence of acute coronary events: prospective cohort study and meta-analysis in 11 European cohorts from the ESCAPE project. BMJ 2014; 348: f7412.

12 Cohen AJ, Brauer M, Burnett R, et al. Estimates and 25-year trends of the global burden of disease attributable to ambient air pollution: an analysis of data from the Global Burden of Diseases Study 2015. Lancet 2017; 389: 1907-1918.

13 Shah AS, Langrish JP, Nair H, et al. Global association of air pollution and heart failure: a systematic review and meta-analysis. Lancet 2013; 382: 1039-1048.

14 Brook RD, Rajagopalan S, Pope CA III, et al. Particulate matter air pollution and cardiovascular disease: an update to the scientific statement from the American Heart Association. Circulation 2010; 121: 2331-2378.

15 Kim H, Kim J, Kim S, et al. Cardiovascular effects of long-term exposure to air pollution: a population-based study with 900845 person-years of follow-up. J Am Heart Assoc 2017; 6: e007170.

16 Park SH, Chen WC, Durmus $\mathrm{N}$, et al. The effects of antigen-specific IgG1 antibody for the pulmonaryhypertension-phenotype and B cells for inflammation in mice exposed to antigen and fine particles from air pollution. PLoS One 2015; 10: $\mathrm{e} 0129910$.

17 Liu J, Ye X, Ji D, et al. Diesel exhaust inhalation exposure induces pulmonary arterial hypertension in mice. Environ Pollut 2018; 237: 747-755.

18 Aung N, Sanghvi MM, Zemrak F, et al. Association between ambient air pollution and cardiac morpho-functional phenotypes. Circulation 2018; 138: 2175-2186.

19 D'Souza JC, Kawut SM, Elkayam LR, et al. Ambient coarse particulate matter and the right ventricle: the Multi-Ethnic Study of Atherosclerosis. Environ Health Perspect 2017; 125: 077019.

20 Galie N, Humbert M, Vachiery JL, et al. 2015 ESC/ERS Guidelines for the diagnosis and treatment of pulmonary hypertension: The Joint Task Force for the Diagnosis and Treatment of Pulmonary Hypertension of the European Society of Cardiology (ESC) and the European Respiratory Society (ERS). Eur Respir J 2015; 46: 903-975.

21 Ho HC, Wong MS, Yang L, et al. Influences of socioeconomic vulnerability and intra-urban air pollution exposure on short-term mortality during extreme dust events. Environ Pollut 2018; 235: 155-162.

22 Hagenaars A, de Vos K, Zaidi MA. Poverty Statistics in the Late 1980s: Research Based on Micro-data. Luxembourg, Eurostat, 1994.

23 de Hoogh K, Gulliver J, Donkelaar AV, et al. Development of West-European $\mathrm{PM}_{2.5}$ and $\mathrm{NO}_{2}$ land use regression models incorporating satellite-derived and chemical transport modelling data. Environ Res 2016; 151: 1-10.

24 Hoeper MM, Kramer T, Pan Z, et al. Mortality in pulmonary arterial hypertension: prediction by the 2015 European pulmonary hypertension guidelines risk stratification model. Eur Respir J 2017; 50: 1700740.

25 Pope CA III, Burnett RT, Thun MJ, et al. Lung cancer, cardiopulmonary mortality, and long-term exposure to fine particulate air pollution. JAMA 2002; 287: 1132-1141.

26 Dockery DW, Stone PH. Cardiovascular risks from fine particulate air pollution. N Engl J Med 2007; 356: 511-513.

27 Sydbom A, Blomberg A, Parnia S, et al. Health effects of diesel exhaust emissions. Eur Respir J 2001; 17: 733-746.

28 Barouki R, Melen E, Herceg Z, et al. Epigenetics as a mechanism linking developmental exposures to long-term toxicity. Environ Int 2018; 114: 77-86.

29 Wei T, Tang M. Biological effects of airborne fine particulate matter (PM2.5) exposure on pulmonary immune system. Environ Toxicol Pharmacol 2018; 60: 195-201.

30 Nemmar A, Hoylaerts MF, Nemery B. Effects of particulate air pollution on hemostasis. Clin Occup Environ Med 2006; 5: 865-881.

31 Adar SD, Sheppard L, Vedal S, et al. Fine particulate air pollution and the progression of carotid intima-medial thickness: a prospective cohort study from the Multi-Ethnic Study of Atherosclerosis and Air Pollution. PLoS Med 2013; 10: e1001430.

32 Aaron CP, Hoffman EA, Hinckley Stukovsky KD, et al. Ambient air pollution and pulmonary vascular structure: the MESA lung and air pollution studies. Am J Respir Crit Care Med 2017; 195: A1746.

33 Calderon-Garciduenas L, Vincent R, Mora-Tiscareno A, et al. Elevated plasma endothelin-1 and pulmonary arterial pressure in children exposed to air pollution. Environ Health Perspect 2007; 115: 1248-1253.

34 Wauters A, Vicenzi M, De Becker B, et al. At high cardiac output, diesel exhaust exposure increases pulmonary vascular resistance and decreases distensibility of pulmonary resistive vessels. Am J Physiol Heart Circ Physiol 2015; 309: H2137-H2144. 
35 Van Hee VC, Adar SD, Szpiro AA, et al. Exposure to traffic and left ventricular mass and function: the Multi-Ethnic Study of Atherosclerosis. Am J Respir Crit Care Med 2009; 179: 827-834.

36 Leary PJ, Kaufman JD, Barr RG, et al. Traffic-related air pollution and the right ventricle. The Multi-Ethnic Study of Atherosclerosis. Am J Respir Crit Care Med 2014; 189: 1093-1100.

37 Aaron CP, Chervona Y, Kawut SM, et al. Particulate matter exposure and cardiopulmonary differences in the Multi-Ethnic Study of Atherosclerosis. Environ Health Perspect 2016; 124: 1166-1173.

38 Vieira JL, Guimaraes GV, de Andre PA, et al. Respiratory filter reduces the cardiovascular effects associated with diesel exhaust exposure: a randomized, prospective, double-blind, controlled study of heart failure: the FILTER-HF Trial. JACC Heart Fail 2016; 4: 55-64.

39 Miller KA, Siscovick DS, Sheppard L, et al. Long-term exposure to air pollution and incidence of cardiovascular events in women. N Engl J Med 2007; 356: 447-458.

40 Di Q, Dai L, Wang Y, et al. Association of short-term exposure to air pollution with mortality in older adults. JAMA 2017; 318: 2446-2456.

41 Ruttens D, Verleden SE, Bijnens EM, et al. An association of particulate air pollution and traffic exposure with mortality after lung transplantation in Europe. Eur Respir J 2017; 49: 1600484.

42 Cromar KR, Gladson LA, Ghazipura M, et al. Estimated excess morbidity and mortality associated with air pollution above American Thoracic Society-recommended standards, 2013-2015. American Thoracic Society and Marron Institute Report. Ann Am Thorac Soc 2018; 15: 542-551.

43 Humbert M, Sitbon O, Chaouat A, et al. Pulmonary arterial hypertension in France: results from a national registry. Am J Respir Crit Care Med 2006; 173: 1023-1030.

44 Lunden MM, Thatcher TL, Hering SV, et al. Use of time- and chemically resolved particulate data to characterize the infiltration of outdoor PM2.5 into a residence in the San Joaquin Valley. Environ Sci Technol 2003; 37: $4724-4732$. 